\title{
NUclei of GAlaxies
}

\section{Radio emission in 7 NUGA sources ${ }^{\star}$}

\author{
M. Krips ${ }^{1,2}$, A. Eckart ${ }^{1}$, T. P. Krichbaum ${ }^{3}$, J.-U. Pott ${ }^{1,4}$, S. Leon $^{5,6}$, R. Neri ${ }^{7}$, S. García-Burillo ${ }^{8}$, \\ F. Combes ${ }^{9}$, F. Boone ${ }^{9}$, A. J. Baker ${ }^{10}$, L. J. Tacconi ${ }^{11}$, E. Schinnerer ${ }^{12}$, and L. K. Hunt ${ }^{13}$ \\ 1 Universität zu Köln, I. Physikalisches Institut, Zülpicher Str. 77, 50937 Köln, Germany \\ e-mail: [krips; eckart] @ph1.uni-koeln.de \\ 2 Harvard-Smithsonian Center for Astrophysics, SMA project, 645 North A'Ohoku Place, Hilo, HI 96720, USA \\ e-mail: mkrips@cfa.harvard.edu \\ 3 Max-Planck-Institut für Radioastronomie, Auf dem Hügel 69, 53121 Bonn, Germany \\ e-mail: tkrichbaum@mpifr-bonn.mpg.de \\ ${ }^{4}$ ESO, Karl-Schwarzschild-Str. 2, 85748 Garching, Germany \\ e-mail: jpott@eso.org \\ 5 Instituto de Astrofísica de Andalucía (CSIC), C/ Camino Bajo de Huétor 24, Apartado 3004, 18080 Granada, Spain \\ ${ }^{6}$ IRAM, Avenida Divina Pastora 7, Núcleo Central, 18012 Granada, Spain \\ e-mail: leon@iram.es \\ 7 Institut de Radio-Astronomie Millimétrique (IRAM), 300 rue de la Piscine, 38406 Saint-Martin-d'Hères, France \\ e-mail: neri@iram.fr \\ 8 Observatorio Astronómico Nacional (OAN)-Observatorio de Madrid, Alfonso XII, 3, 28014 Madrid, Spain \\ e-mail: s.gburillo@oan.es \\ 9 Observatoire de Paris, LERMA, 61 Av. de l'Observatoire, 75014 Paris, France \\ e-mail: francoise.combes@obspm.fr; fboone@mpifr-bonn.mpg.de \\ 10 Department of Physics and Astronomy, Rutgers, the State University of New Jersey, 136 Frelinghuysen Road, Piscataway, \\ NJ 08854-8019, USA \\ e-mail: ajbaker@physics.rutgers.edu \\ 11 Max-Planck-Institut für Extraterrestrische Physik, Postfach 1312, 85741 Garching, Germany \\ e-mail: linda@mpe.mpg.de \\ 12 Max-Planck-Institut für Astronomie, Königstuhl 17, 69117 Heidelberg, Germany \\ e-mail: schinner@mpia.de \\ 13 INAF-Istituto di Radioastronomia/Sezione Firenze, Largo E. Fermi 5, 50125 Firenze, Italy \\ e-mail: hunt@arcetri.astro.it
}

Received 17 February 2006 / Accepted 21 December 2006

\section{ABSTRACT}

\begin{abstract}
We present high angular resolution radio snap-shot observations of seven nearby low-luminosity active galaxies (LLAGN) from the NUclei of GAlaxies (NUGA) survey. The observations were conducted with MERLIN and EVN/VLBI at $18 \mathrm{~cm}$ and $6 \mathrm{~cm}$. At all observed angular resolutions and frequencies, we find indications for extended emission in about $\sim 40 \%$ of the sources, consistent with the decrease of flux with increasing angular resolution. The extended components resemble jet emission in a majority of cases, consistent with the optically thin synchrotron emission implied by their steep spectra. We consider the compact $6 \mathrm{~cm}$ EVN/VLBI radio emission of our sources in the context of the "fundamental plane" that previous LLAGN studies identified within the threedimensional parameter space of radio luminosity, X-ray luminosity, and black hole mass. We demonstrate, using NGC 7217 and NGC 1068 as particular examples, that high-resolution, multi-epoch radio observations offer useful information about the origin of offsets from the fundamental plane.
\end{abstract}

Key words. galaxies: active - galaxies: Seyfert - galaxies: nuclei - galaxies: jets - radio continuum: galaxies

\section{Introduction}

Nuclear activity in galaxies is detected at many different levels, supporting the idea that it reflects different phases in galaxy

\footnotetext{
* EVN: The European VLBI Network is a joint facility of European, Chinese, South African and other radio astronomy institutes funded by their national research councils. MERLIN is a national facility operated by the University of Manchester on behalf of PPARC. VLBI including the VLBA: The National Radio Astronomy Observatory is a facility of the National Science Foundation operated under cooperative agreement by Associated Universities, Inc.
}

evolution, i.e., that every galaxy might exhibit nuclear activity at some point in its life. Nuclear activity is thought to be due to accretion onto supermassive black holes (SMBHs), which are now accepted to reside in virtually all galaxies, at least those with bulges (Kormendy \& Richstone 1995; Magorrian et al. 1998). The tight correlation of SMBH mass with the stellar velocity dispersion in the bulge (Magorrian et al. 1998; Richstone et al. 1998; Gebhardt et al. 2000; Ferrarese \& Merritt 2000; Tremaine et al. 2002; Shields et al. 2003; Marconi \& Hunt 2003) along with the similar redshift peaks $(z \approx 2)$ of the quasar and starburst 
Table 1. Basic properties of the NUGA sources taken from NED. ${ }^{a} i \equiv$ inclination of the galaxy. $\mathrm{H}=\mathrm{HII}-$ region, $\mathrm{S}=\mathrm{Seyfert}$ and $\mathrm{L}=\mathrm{LINER}$.

\begin{tabular}{lcccccc}
\hline \hline Name & $\begin{array}{c}\text { RA } \\
(\mathrm{J} 2000)\end{array}$ & $\begin{array}{c}\text { Dec } \\
(\mathrm{J} 2000)\end{array}$ & $\begin{array}{c}v_{\text {hel }} \\
{\left[\mathrm{km} \mathrm{s}^{-1}\right]}\end{array}$ & $\begin{array}{c}i^{a} \\
\left({ }^{\circ}\right)\end{array}$ & $\begin{array}{c}\text { Host } \\
\text { type }\end{array}$ & $\begin{array}{c}\text { Nuclear } \\
\text { type }\end{array}$ \\
\hline NGC 1961 & $05: 42: 04.6$ & $69: 22: 43.0$ & 3934 & 49 & SAB(rs)c & L2 \\
NGC 2782 & $09: 14: 05.1$ & $40: 06: 49.0$ & 2562 & 42 & SAB(rs) pec & H \\
NGC 3147 & $10: 16: 53.6$ & $73: 24: 03.0$ & 2820 & 26 & SA(rs)bc & S2 \\
NGC 3718 & $11: 32: 35.0$ & $53: 04: 04.0$ & 994 & 60 & pec & L1.9 \\
NGC 4579 & $12: 37: 43.4$ & $11: 49: 05.0$ & 1519 & 37 & SAB(rs)b & S1.9/L1.9 \\
NGC 5953 & $15: 34: 32.4$ & $15: 11: 38.0$ & 1965 & 36 & SAa pec & S2 \\
NGC 7217 & $22: 07: 52.4$ & $31: 21: 34.0$ & 952 & 35 & $($ R)SAB(rs)a & L2 \\
\hline
\end{tabular}

populations, suggests a connected, or at least similar evolution between starburst and SMBH activity. As implied by the population peak, nuclear activity seems to increase with redshift, leaving predominantly Low-Luminosity AGN (LLAGN) and inactive galaxies in the nearby universe. However, many similarities are found between LLAGN and High-Luminosity AGN (HLAGN) over the entire electromagnetic spectrum, further indicating that different activity phases might simply correspond to different evolutionary stages.

Recent studies strongly suggest that HLAGN, LLAGN, and even X-ray binaries occupy a so-called "fundamental plane" within the three-dimensional parameter space of central black hole mass vs. radio luminosity vs. X-ray luminosity (Merloni et al. 2003; Falcke et al. 2004). This strongly indicates common underlying processes. The exact origin of this correlation is still the subject of controversy, although it is now widely accepted that different accretion rates and/or different accretion efficiencies certainly play a significant role in setting the various activity levels. In this picture, LLAGN may be driven by low accretion rates into a mode in which they only release a small fraction of their energy through radiation from a radiatively inefficient accretion flow (RIAF); the remainder is instead converted into thermal, turbulent, or mechanical energy. HLAGN, however, efficiently cool through radiation, thus producing a high activity level. A variety of RIAF models have been invoked over the past decade, including Bondi-Hoyle accretion (Melia \& Falcke 2001), Advection Dominated Accretion Flows (ADAFs; e.g., Narayan et al. 1995), Convection Dominated Accretion Flows (CDAFs; Ball et al. 2001), ADiabatic Inflow Outflow Solutions (ADIOS; Blandford \& Begelman 1999) and quasimono-energetic electron distributions (Beckert \& Duschl 1997). However, ADAFs and Bondi-Hoyle accretion are found to fail for $\operatorname{Sgr} A^{\star}$ in the center of our own galaxy, since they entail gas densities which are significantly higher than observed; they are thus already regarded as rather out-of-date ${ }^{1}$.

As an alternative to RIAFs, a disk-jet coupling can also produce the observed fundamental plane (Heinz \& Sunyaev 2003); here, the base of the jet rather than a RIAF/disk produces the compact (optically thick synchrotron) emission in LLAGN, while the accretion disk dominates the emission in HLAGN (e.g., Falcke \& Markoff 2000). Previous studies of LLAGN (e.g., Ulvestad \& Wilson 1984; Pedlar et al. 1993; Falcke et al. 2000; Nagar et al. 2002, 2005; Anderson et al. 2004; Anderson \& Ulvestad 2005) indicate radio jets and/or compact radio emission similar to but less powerful than in HLAGN. Jets are intimately connected to accretion onto compact objects (e.g., Pringle 1993; Blandford 1993) and are thus regarded as a clear signature of accretion-powered AGN. However, it is impossible at the

${ }^{1}$ For a more detailed description of the different RIAFs we refer to Quataert et al. (2003), Eckart et al. (2004) and the references given above. moment to distinguish between emission powered by a jet and a RIAF, so various combinations of the two must generally be considered (e.g., Yuan et al. 2002). In each case, high angular resolution observations of the radio emission offer substantial insight into the nature of the underlying process.

The seven sources studied in this paper belong to the IRAM NU(clei of) GA(laxies) survey (García-Burillo et al. 2003). The NUGA project aims at studying the molecular gas distributions and kinematics with the highest currently available angular resolution and sensitivity in a sample of 12 nearby Seyfert and LINER galaxies and transition objects (García-Burillo et al. 2003; Combes et al. 2004; Krips et al. 2005; García-Burillo et al. 2005). The study of radio emission is an ideal complement to the molecular gas analysis in investigating the activity in these sources. We thus selected those sources in our sample with existing and clear VLA radio detections (Becker et al. 1995; Condon et al. 1996; Ho \& Ulvestad 1999) for a higher angular resolution study with MERLIN and VLBI.

The paper is organized in the following way. Section 2 gives an overview of the observations. Section 3 describes the obtained results. The nature of the detected radio emission is discussed in Sect. 4. A comparison of the radio luminosities with respect to the fundamental plane is presented in Sect. 5. Finally, the paper ends with a summary and conclusions in Sect. 6.

\section{Observations}

Radio snapshot ${ }^{2}$ observations of seven NUGA sources (Table 1) have been carried out at $18 \mathrm{~cm}$ and $6 \mathrm{~cm}$ using MERLIN and EVN/VLBI between 2001 and 2004. Two sources from this sample (NGC 3718 and NGC 3147) have been used for deeper integration (6-9 h per source). The history of the observations is listed in Table 2. We selected these seven sources from the NUGA survey because they have all been clearly detected on arcsecond scales before (VLA observations: Becker et al. 1995; Condon et al. 1996; Ho \& Ulvestad 1999) but lacked consistent mapping at higher angular resolution. With both MERLIN and EVN/VLBI, we took advantage of the phase-reference technique (Beasley \& Conway 1995) by observing alternately the target and a phase calibrator located within a few degrees. The EVN/VLBI observations were conducted with the Mark IV system at a rate of $256 \mathrm{Mb} \mathrm{s}^{-1}$ using $8 \mathrm{IFs}$, each of $8 \mathrm{MHz}$ bandwidth, and 2-bit sampling (mode-256-8-2). The sources were observed in LCP. At MERLIN, polarization measurements were also carried out but without any significant detection. The visibility amplitudes were calibrated assuming Kelvin to Jansky conversion factors, gain-elevation dependencies, and system temperatures measured during the observations. This and careful amplitude self-calibration using the available closure amplitudes provided a typical calibration accuracy of 5-10\%. Approximate

\footnotetext{
${ }^{2}$ Snapshot corresponds here to $\sim 3-5 \mathrm{~h}$ on-source integration time.
} 
beam sizes range from 0.05 to $0.5^{\prime \prime}$ for MERLIN and 0.001 to 0.04 " for EVN. RMS image noises range from $\sim 1 \mu \mathrm{Jy}$ to $\sim 1 \mathrm{mJy}^{3}$. All data were reduced with the AIPS package and mapped either in AIPS itself or using DIFMAP (van Moorsel et al. 1996; Shepherd 1997).

Additionally, we have information on the continuum emission at $3 \mathrm{~mm}$ and $1 \mathrm{~mm}$ for all seven sources, which was gained during the $\mathrm{CO}(1-0)$ and $\mathrm{CO}(2-1)$ observations of the NUGA sample at the IRAM Plateau de Bure Interferometer (PdBI). For a more detailed description of the PdBI observations, we refer the reader to previous NUGA publications (e.g., García-Burillo et al. 2003; Combes et al. 2004; Krips et al. 2005; García-Burillo et al. 2005).

\section{Results}

We fitted Gaussian profiles in the image plane to all of our radio maps to get estimates of component sizes (by using the fitted FWHM of the continuum emission and half the beamsize as a cutoff lower limit) and to derive the respective fluxes (Tables 3 and 4) and positions (Table 3). We first start with some general results for the entire sample in Sect. 3.1 and then discuss each galaxy individually in Sect. 3.2.

\subsection{General results}

Tables 3 and 4 list the derived positions, fluxes and sizes of the respective sources. The position of each component is consistent across all data sets. Figures 1 to 3 show the continuum maps of the seven sources for each frequency and instrument. We find extended emission on all angular scales. This is confirmed by comparing the peak intensities and integrated flux densities with each other (a difference indicating resolved emission). Extended emission is also implied by the decrease of flux density as we move from VLA to MERLIN to EVN resolution at a fixed frequency (e.g., in NGC 1961 and NGC 7217; see Table 7 for the VLA luminosities). In at least three objects (NGC 2782, NGC 5953, and NGC 7217), we see clear signs of a jet on MERLIN scales, and in another two (NGC 1961 and NGC 3718) we find indications of jet-like morphologies. Interestingly, while for the stronger sources we find flat to inverted spectra, the weaker sources have steep spectra (Fig. 4 and Table 5).

\subsection{Results on individual galaxies}

In the next subsections we will present separately the individual results for each galaxy.

We determine for all galaxies the nuclear bolometric luminosities via the X-ray flux unless otherwise stated. Ulvestad \& Ho (2001) propose an empirical relation to estimate the bolometric luminosity of the nucleus in active galaxies. Based on a sample of 10 objects, they find $L_{\mathrm{bol}}=6.7 \times L_{\mathrm{x}}(2-10 \mathrm{keV})$. Assuming this equation and taking the X-ray luminosity (Table 7) published by Roberts \& Warwick (2000), Ulvestad \& Ho (2001), Fabbiano et al. (1992), or Terashima et al. (2002), we can crudely estimate the nuclear bolometric luminosity for each galaxy. In some cases, this estimate should be taken as an upper limit: particularly for Roberts \& Warwick (2000), a significant contribution of circumnuclear star formation to the X-ray flux cannot be entirely excluded, due to the low $\left(\sim 10^{\prime \prime}\right)$ angular resolution of

3 The lower rms noise corresponds to the two deep integrations, not the snap-shot observations. the observations. Furthermore, we have hard $(2-10 \mathrm{keV}) \mathrm{X}$-ray luminosities for only three sources, requiring that we extrapolate the hard X-ray flux from the soft $0.2-4 \mathrm{keV}$ ( or $0.2-2 \mathrm{keV}$ ) band for the remaining sources as a crude estimate. These values are only regarded as rough estimates of the nuclear hard X-ray luminosities, given significant uncertainties in the contribution of emission from the host galaxy, the spectral shape in this band (sometimes a break occurs at $\sim 1 \mathrm{keV}$ ), and the amount of absorption. The last-mentioned uncertainty might be the most important one, as all of the sources studied in this paper are classified as type 2 Seyfert or LINER, implying an obscuration of the central engine in the standard AGN unification paradigm. However, previous X-ray studies of type 2 Seyfert and LINER galaxies, including NGC 3147, NGC 4579, and NGC 7217 (e.g., Terashima et al. 2002), indicate that even these type 2 LLAGN typically show spectral indices of $\Gamma \simeq 1.6-2.0$ (with $P(E)=A \cdot E^{-\Gamma}$ ), suggesting that our approximation $(\Gamma \geq 1)$ might give a reasonable and reliable upper limit.

We estimate black hole masses for all galaxies using the $M_{\mathrm{bh}}-\sigma_{\mathrm{s}}$ correlation, with $\sigma_{\mathrm{s}}$ being the stellar velocity dispersion of the bulge component (e.g., Gebhardt et al. 2000; Tremaine et al. 2002). The velocity dispersion has been taken from McElroy et al. (1995) for almost all galaxies except for NGC 3718, for which we estimated it from FWHM(N [II]) taken from Ho et al. (1997; see also caption of Table 7). As a caveat, one has to keep in mind that the $M_{\mathrm{bh}}-\sigma_{\mathrm{s}}$ correlation has been mostly established for elliptical galaxies, while our sample is based on late type spiral galaxies. However, recent results from reverberation mapping indicate that the relation still holds for spiral galaxies containing a Seyfert nucleus (e.g., Wandel et al. 1999; Kaspi et al. 2000; Nelson et al. 2004; Onken et al. 2004; Peterson et al. 2005). A different, recently significantly improved approach to determine the black hole mass is the bulge-to-blackhole mass correlation (e.g., Marconi \& Hunt 2003; Häring \& Rix 2004). An estimate of the bulge masses for the NUGA sample is in preparation (Hunt et al.) so that we refer the reader to this paper, which will also include a comparison of the various black hole mass estimates.

\subsubsection{NGC 1961}

NGC 1961 contains a LINER type 2 nucleus in an SAB(rs)c host galaxy at a distance of $52 \mathrm{Mpc}$. This is the most distant source in our survey. Assuming $\sigma_{\mathrm{s}}=255 \mathrm{~km} \mathrm{~s}^{-1}$ (McElroy 1995), $M_{\mathrm{bh}}$ is inferred to be $3.0 \times 10^{8} \mathcal{M}_{\odot}$ (Table 7). Taking $L_{\mathrm{x}} \simeq 4 \times 10^{40} \mathrm{erg} \mathrm{s}^{-1}$ (Roberts \& Warwick 2000), the nuclear bolometric luminosity of NGC 1961 can be estimated as $\sim 10^{41} \mathrm{erg} \mathrm{s}^{-1}$ implying a subEddington $^{4}$ system with $L_{\mathrm{bol}} / L_{\mathrm{ed}} \simeq 10^{-6}-10^{-5}$ (Table 7).

The MERLIN $18 \mathrm{~cm}$ map clearly shows extended emission, consistent with the difference between peak and integrated flux densities (see Fig. 1 and Table 4) and previous VLA maps which also indicate extended emission (Condon et al. 1995). The deconvolved size is slightly bigger than the beam size, giving further evidence for extended emission. A two-component Gaussian fit assuming a core and a "jet-like" feature results in a $10 \sigma$ determination of the core flux but only in a $\sim 2 \sigma$ estimate for the flux of the potential jet. The MERLIN $6 \mathrm{~cm}$ map independently indicates extended emission and, besides a compact core, three additional components. The northeastern and the southeastern features lie in the direction of the extensions seen in the MERLIN $18 \mathrm{~cm}$ map. However, given that the southwestern feature appears to be symmetrically positioned on the other side of

\footnotetext{
${ }^{4} L_{\text {ed }} / L_{\odot}=3.22 \times 10^{4} M / M_{\odot}$.
} 
Table 2. Radio observations for seven NUGA sources. ${ }^{a}$ NGC 4579 was excluded since MERLIN observations were already carried out; the data are taken from the MERLIN archive. ${ }^{b}$ NGC 3147 and NGC 4579 were not observed because VLBA data were already available from Ulvestad \& Ho (2001). ${ }^{c}$ The first observations in 2002 were not successful due to technical problems with some of the antennas and were thus repeated in 2003; the results of both observations are consistent with each other but only the 2003 data sets are used for further discussion due to the higher quality of the data. ${ }^{d} \mathrm{~Eb} \equiv$ Effelsberg, $\mathrm{Jb} \equiv$ Jodrell Bank, Wb $\equiv$ Westerbork, Mc $\equiv$ Medicina, $\mathrm{Nt} \equiv \mathrm{Noto}$, On85 $\equiv$ Onsala $25 \mathrm{~m}, \mathrm{Sh} \equiv \mathrm{Shanghai}$, $\mathrm{Ur} \equiv$ Urumqi, $\mathrm{Tr} \equiv$ Torun (see EVN homepage for more information on the respective telescopes: http://www . evlbi .org/).

\begin{tabular}{|c|c|c|c|c|}
\hline Instrument & Obs. frequency & Month Year & VLBI stations $^{d}$ & Sources \\
\hline MERLIN & $1.66 \mathrm{GHz}$ & $2001 / 2002$ & - & all except NGC $4579^{a}$ \\
\hline EVN & $1.63 \mathrm{GHz}$ & February 2002 & $\begin{array}{l}\mathrm{Eb}, \mathrm{Jb}, \mathrm{Wb}, \mathrm{Mc}, \mathrm{Nt} \\
\text { On85, Sh, Ur, Tr }\end{array}$ & NGC 3147, NGC 3718 \\
\hline $\mathrm{EVN}$ & $1.63 \mathrm{GHz}$ & November (2002) $2003^{c}$ & $\begin{array}{c}\mathrm{Eb}, \mathrm{Jb}, \mathrm{Wb}, \mathrm{Mc}, \mathrm{Nt} \\
\text { On85, Sh, Ur, Tr }\end{array}$ & $\begin{array}{l}\text { NGC 1961, NGC 2782, NGC } 4579 \text {, } \\
\text { NGC 5953, NGC } 7217\end{array}$ \\
\hline MERLIN & $4.99 \mathrm{GHz}$ & $2002 / 2003$ & - & all \\
\hline EVN & $4.99 \mathrm{GHz}$ & June 2003 & $\begin{array}{l}\mathrm{Eb}, \mathrm{Jb}, \mathrm{Wb}, \mathrm{Mc}, \mathrm{Nt} \\
\text { On85, Sh, Ur, Tr }\end{array}$ & NGC 3147, NGC 3718 \\
\hline global VLBI & $4.99 \mathrm{GHz}^{b}$ & May 2004 & $\begin{array}{c}\text { Eb, Jb, Wb, Mc, Nt, } \\
\text { On85, Sh, Ur, Tr, VLBA }\end{array}$ & $\begin{array}{l}\text { NGC 1961, NGC 2782, NGC 3718, } \\
\text { NGC 5953, NGC } 7217\end{array}$ \\
\hline
\end{tabular}

Table 3. Radio positions of the seven NUGA sources, mainly derived from the EVN $6 \mathrm{~cm}$ (core) or MERLIN $18 \mathrm{~cm} / 6 \mathrm{~cm}$ (jet) observations by fitting Gaussian profiles to the emission (the positions are coincident within the uncertainties of the different observations). The positional uncertainties are estimated from the Gaussian fits and also include the most recent (i.e., determined in the past 4 years) astromectric errors of the used phase-calibrators.

\begin{tabular}{lllll}
\hline \hline Name & RA [h:m:s] & $\Delta \alpha[\mathrm{s}]$ & Dec $\left[{ }^{\circ}::^{\prime \prime}\right]$ & $\Delta \delta\left[^{\prime \prime}\right]$ \\
\hline NGC 1961-core & $05: 42: 04.6477$ & \pm 0.0003 & $69: 22: 42.375$ & \pm 0.004 \\
NGC 2782-core & $09: 14: 05.1124$ & \pm 0.0003 & $40: 06: 49.316$ & \pm 0.004 \\
NGC 2782-jet & $09: 14: 05.105$ & \pm 0.001 & $40: 06: 49.27$ & \pm 0.01 \\
NGC 3147-core & $10: 16: 53.6500$ & \pm 0.0003 & $73: 24: 02.680$ & \pm 0.004 \\
NGC 3718-core & $11: 32: 34.8530$ & \pm 0.0003 & $53: 04: 04.518$ & \pm 0.004 \\
NGC 3718-jet & $11: 32: 34.854$ & \pm 0.001 & $53: 04: 04.523$ & \pm 0.004 \\
NGC 4579-core & $12: 37: 43.522$ & \pm 0.001 & $11: 49: 05.498$ & \pm 0.004 \\
NGC 5953-core & $15: 34: 32.383$ & \pm 0.002 & $15: 11: 37.59$ & \pm 0.02 \\
NGC 5953-jet & $15: 34: 32.390$ & \pm 0.003 & $15: 11: 38.03$ & \pm 0.02 \\
NGC 7217-core & $22: 07: 52.3933$ & \pm 0.0003 & $31: 21: 33.646$ & \pm 0.004 \\
\hline
\end{tabular}

the core with respect to the northeastern feature, sidelobe effects cannot be totally excluded for these two components. Also, since the signal-to-noise ratios of all three "off-core" components are still marginal, they remain questionable. We find indications of extended emission in the EVN $18 \mathrm{~cm}$ data as well by comparing the peak with the integrated flux density. The distribution appears to be slightly resolved and shows an elongation to the northeast but with a different PA than found in the MERLIN maps. The extensions are in the direction of the beam, so we must again consider sidelobe effects. The two artificial components to the west and to the east are found to be at the positions of the sidelobes. The $6 \mathrm{~cm}$ EVN component is only marginally resolved and the deconvolved size is smaller than the beam size (by a factor of $\sim 2$ ). The PdBI $3 \mathrm{~mm}$ map also reveals a jet-like component of $\sim 1 \mathrm{mJy}$ which is $\sim 4^{\prime \prime}$ northeast of the nucleus (Baker et al. in prep.). Further radio observations are needed to clarify the different orientations of the jet-like components found at different wavelengths and angular resolution.

Both EVN and MERLIN data imply a spectral index of $\alpha=-0.3$ (for $f_{v} \propto v^{\alpha}$ ), indicating a decrease of flux with decreasing wavelength. The higher resolutions of the $6 \mathrm{~cm}$ maps mean that the spectral index might be higher and so the spectrum even flatter. The upper limit on the spectral index derived from the PdBI fluxes is similar to the cm-derived values and also suggests a flat spectrum.

\subsubsection{NGC 2782}

The SAB(rs)pec host galaxy of NGC 2782 ( $D=35 \mathrm{Mpc}$ ) harbours a dominant nuclear HII region; historically, its nuclear activity has been supposed to be mainly dominated by a powerful nuclear starburst rather than by an AGN. However, recent observations of water maser emission in this object suggest that a hidden AGN might be present as well (Braatz et al. 2004). Its central black hole mass can be estimated as $4 \times 10^{7} \mathcal{M}_{\odot}$ assuming $\sigma_{\mathrm{s}}=146 \mathrm{~km} \mathrm{~s}^{-1}$ (McElroy 1995). The nuclear bolometric luminosity amounts to $\sim 10^{41} \mathrm{erg} \mathrm{s}^{-1}$ assuming $L_{\mathrm{X}}$ from Table 7. A comparison with the Eddington luminosity indicates that NGC 2782 might also radiate at a sub-Eddington rate with $L_{\text {bol }} / L_{\text {ed }} \simeq 10^{-5}-10^{-4}$.

A strong core and a strong extended jet are visible in both the MERLIN $18 \mathrm{~cm}$ and $6 \mathrm{~cm}$ maps (see Fig. 1 and Table 4), supporting the presence of an optically hidden AGN; VLA maps also show extended and diffuse emission on arcsecond scales (Saikia et al. 1994). The jet is elongated towards the south/southeast and has a length of $\sim 0.3^{\prime \prime}(=53 \mathrm{pc})$. The core seems to be slightly extended, too. Going to $6 \mathrm{~cm}$ and thus to higher angular resolution, the core and the jet become more resolved, as indicated also by their lower fluxes. The positions of both components agree at the two wavelengths. The EVN $18 \mathrm{~cm}$ maps also show an extended component, again consistent with the different peak and integrated flux densities. The extension is not in the direction of the beam. However, the PA of the extended emission is slightly different from that in the MERLIN maps, raising doubts about its reliability. In the EVN $6 \mathrm{~cm}$ map, we find a $\sim 3 \sigma$ peak at the position of the nucleus. Resolution effects may play a role for this tentative detection. The spectral index derived from the MERLIN data agrees with the upper limit obtained from the EVN data. Parts of the jets might affect the core emission in the MERLIN maps since core and jet emission cannot be easily separated from each other in the $18 \mathrm{~cm}$ MERLIN map, complicating the estimate of the spectral index for the core and the jet.

No continuum emission was detected at $3 \mathrm{~mm}$ or $1 \mathrm{~mm}$ with the IRAM PdBI. The derived upper limits are given in Table 4. 
Table 4. Results of the radio and mm continuum observations with MERLIN, EVN and PdBI. ${ }^{a}$ From the MERLIN archive (PI: N.Nagar). ${ }^{b}$ Taken from VLBA observation carried out by Ulvestad \& Ho (2001; $\equiv$ Ulv01). Please note that the flux errors do not include the calibration uncertainties of 5-10\% and represent only the statistical uncertainties of the fit. The given angles are derived from North to East.

\begin{tabular}{|c|c|c|c|c|c|c|}
\hline \multirow[b]{3}{*}{ Name } & \multirow{2}{*}{\multicolumn{6}{|c|}{ MERLIN }} \\
\hline & & & & & & \\
\hline & $\begin{array}{c}\text { Peak } \\
\text { flux } \\
{[\text { mJy/beam] }}\end{array}$ & $\begin{array}{c}\text { Integrated } \\
\text { flux density } \\
{[\mathrm{mJy}]}\end{array}$ & $\begin{array}{c}\text { Deconvolved } \\
\text { size } \\
\text { " } x^{\prime \prime} @{ }^{\circ}\end{array}$ & $\begin{array}{c}\text { Peak } \\
\text { flux } \\
{[\mathrm{mJy} / \text { beam] }}\end{array}$ & $\begin{array}{c}\text { Integrated } \\
\text { flux density } \\
{[\mathrm{mJy}]}\end{array}$ & $\begin{array}{c}\text { Deconvolved } \\
\text { size } \\
" \prime \times^{\prime \prime} @^{\circ}\end{array}$ \\
\hline NGC 1961-core & $2.2 \pm 0.2$ & $3.3 \pm 0.4$ & $0.1 \times 0.09 @ 143$ & $1.2 \pm 0.3$ & $1.8 \pm 0.6$ & $0.1 \times 0.1$ \\
\hline NGC 2782-core & $1.4 \pm 0.1$ & $4.6 \pm 0.6$ & $0.2 \times 0.1 @ 20$ & $0.7 \pm 0.2$ & $2.5 \pm 0.7$ & 0.1×0.06@153 \\
\hline NGC 2782-jet & $0.3 \pm 0.1$ & $1.8 \pm 1.0$ & $0.3 \times 0.2 @ 74$ & $0.6 \pm 0.2$ & $1.4 \pm 0.5$ & 0.08×0.06@145 \\
\hline NGC 3147-core & $7.4 \pm 0.2$ & $8.6 \pm 0.3$ & 0.06×0.04@150 & $10.2 \pm 0.1$ & $10.0 \pm 0.1$ & $0.03 \times 0.03$ \\
\hline NGC 3718-core & $1.2 \pm 0.1$ & $4.0 \pm 0.6$ & $0.4 \times 0.1 @ 150$ & $5.3 \pm 0.1$ & $6.1 \pm 0.3$ & 0.05×0.03@139 \\
\hline NGC 3718-jet & $4.7 \pm 0.2$ & $4.5 \pm 0.3$ & 0.05×0.03@70 & - & - & - \\
\hline NGC 4579-core & $5.0^{a}$ & - & - & $15.2 \pm 0.2$ & $17.2 \pm 0.3$ & 0.09×0.03@146 \\
\hline NGC 5953-core & $1.6 \pm 0.2$ & $1.5 \pm 0.3$ & $0.03 \times 0.03$ & $0.5 \pm 0.1$ & $0.4 \pm 0.2$ & 0.05×0.03@67 \\
\hline NGC 5953-jet & $1.2 \pm 0.2$ & $1.2 \pm 0.3$ & 0.3×0.2@10 & - & - & - \\
\hline NGC 7217-core & $1.5 \pm 0.1$ & $2.6 \pm 0.3$ & $0.2 \times 0.1 @ 157$ & $5.0 \pm 0.5$ & $5.0 \pm 0.5$ & 0.04×0.03@13 \\
\hline \multirow[b]{2}{*}{ Name } & \multicolumn{6}{|c|}{ EVN/VLBI } \\
\hline & $\begin{array}{c}\text { Peak } \\
\text { flux } \\
{[\mathrm{mJy} / \text { beam }]}\end{array}$ & $\begin{array}{c}\text { Integrated } \\
\text { flux density } \\
{[\mathrm{mJy}]}\end{array}$ & $\begin{array}{c}\text { Deconvolved } \\
\text { size } \\
\text { " } \times^{\prime \prime} @{ }^{\circ}\end{array}$ & $\begin{array}{c}\text { Peak } \\
\text { flux } \\
{[\mathrm{mJy} / \mathrm{beam}]}\end{array}$ & $\begin{array}{c}\text { Integrated } \\
\text { flux density } \\
{[\mathrm{mJy}]}\end{array}$ & $\begin{array}{c}\text { Deconvolved } \\
\text { size } \\
"{ }^{\prime \prime} @^{\circ}\end{array}$ \\
\hline NGC 1961-core & $0.50 \pm 0.07$ & $1.2 \pm 0.2$ & $0.05 \times 0.01 @ 21$ & $0.73 \pm 0.05$ & $0.9 \pm 0.1$ & $0.004 \times 0.003 @ 55$ \\
\hline NGC 2782-core & $0.40 \pm 0.06$ & $1.2 \pm 0.2$ & $0.05 \times 0.01 @ 170$ & $\leq 0.4$ & - & - \\
\hline NGC 3147-core & $6.1 \pm 0.1$ & $6.0 \pm 0.2$ & $0.004 \times 0.004$ & $10.1 \pm 0.3$ & $9.3 \pm 0.5$ & $0.006 \times 0.006$ \\
\hline NGC 3718-core & $4.8 \pm 0.1$ & $4.7 \pm 0.3$ & $0.008 \times 0.007 @ 173$ & $5.6 \pm 0.1$ & $7.1 \pm 0.2$ & $0.006 \times 0.005 @ 115$ \\
\hline NGC 4579-core & $17.8 \pm 0.6$ & $20.7 \pm 1.0$ & $0.02 \times 0.01 @ 4$ & - & - & - \\
\hline NGC 4579 (Ulv01) ${ }^{b}$ & - & 18.3 & - & - & 22.8 & - \\
\hline NGC 5953-core & $\leq 0.3$ & - & - & $\leq 0.4$ & - & - \\
\hline NGC 7217-core & $0.5 \pm 0.1$ & $0.5 \pm 0.1$ & $0.03 \times 0.02 @ 71$ & $0.41 \pm 0.06$ & $1.2 \pm 0.2$ & 0.005×0.003@106 \\
\hline \multirow[b]{2}{*}{ Name } & \multicolumn{6}{|c|}{ PdBI } \\
\hline & $\begin{array}{c}\text { Peak } \\
\text { flux } \\
{[\mathrm{mJy} / \text { beam }]}\end{array}$ & $\begin{array}{c}\text { Integrated } \\
\text { flux density } \\
{[\mathrm{mJy}]}\end{array}$ & $\begin{array}{c}\text { Size } \\
" \times^{\prime \prime} @ \circ\end{array}$ & $\begin{array}{c}\text { Peak } \\
\text { flux } \\
{[\mathrm{mJy} / \mathrm{beam}]}\end{array}$ & $\begin{array}{c}\text { Integrated } \\
\text { flux density } \\
{[\mathrm{mJy}]}\end{array}$ & $\begin{array}{c}\text { Size } \\
\text { " } x^{\prime \prime} @ \circ\end{array}$ \\
\hline NGC 1961 & $2.6 \pm 0.4$ & $3 \pm 0.3$ & $3.5 \times 1.8 @ 57$ & $\leq 1.5(3 \sigma)$ & - & - \\
\hline NGC 2782 & $\leq 1.0(3 \sigma)$ & - & - & $\leq 3.0(3 \sigma)$ & - & - \\
\hline NGC 3147 & $5.3 \pm 0.3$ & $5.0 \pm 0.3$ & $1 \times 1$ & $2.8 \pm 0.5$ & $4.0 \pm 0.4$ & $1 \times 1$ \\
\hline NGC 3718 & $10 \pm 2$ & $11 \pm 2$ & $2 \times 2$ & $9.5 \pm 0.7$ & $14 \pm 0.8$ & $1 \times 1$ \\
\hline NGC 4579 & $11.3 \pm 0.5$ & $11 \pm 0.4$ & $0.5 \times 0.5$ & $12 \pm 2$ & $11 \pm 2$ & $0.5 \times 0.5$ \\
\hline NGC 5953 & $\leq 2(3 \sigma)$ & - & - & $\leq 4(3 \sigma)$ & - & - \\
\hline NGC 7217 & $\leq 1.5(3 \sigma)$ & - & - & $\leq 4(3 \sigma)$ & - & - \\
\hline
\end{tabular}

\subsubsection{NGC 3147}

NGC 3147 is classified as SA(rs)bc galaxy at a distance of $38 \mathrm{Mpc}$ with a Seyfert type 2 nucleus. The central black hole mass can be derived as $\sim 4 \times 10^{8} \mathcal{M}_{\odot}$ with $\sigma_{\mathrm{s}}=268 \mathrm{~km} \mathrm{~s}^{-1}$ (McElroy et al. 1995) and the nuclear bolometric luminosity is $\sim 2 \times 10^{42} \mathrm{erg} \mathrm{s}^{-1}$ (Table 7), implying $L_{\mathrm{bol}} / L_{\mathrm{ed}} \simeq 5 \times 10^{-5}$. Therefore, NGC 3147 radiates at a sub-Eddington rate.

NGC 3147 is among the strongest radio sources in our survey (see Fig. 1 and Table 4). In all maps (including the mm-data and VLA data (Ho \& Ulvestad 1999), it appears to be pointlike. The almost identical fluxes determined at both wavelengths and instruments are a further sign of very compact emission in this object. The flat to inverted spectral index derived from the EVN/MERLIN flux densities indicates that no extended jet is present. The point-like structure and the spectral indices (at $\mathrm{cm}$ wavelengths) are consistent with what Ulvestad \& Ho (2002) found in VLBA data (see also Anderson et al. 2004; Anderson \& Ulvestad 2005). Our two observing epochs for the EVN $18 \mathrm{~cm}$ data show no evidence for any variability of this source. We find a turnover between the cm- and the mm-fluxes (Fig. 4), as can also be seen in the different spectral indices at $\mathrm{mm}$ - and cmwavelengths (Table 5). The turnover is most likely caused by synchrotron self absorption, as is discussed in further detail in a separate paper (Krips et al. 2006).

\subsubsection{NGC 3718}

The galaxy NGC 3718 is located at a distance of $13 \mathrm{Mpc}$, hosts a LINER type 1.9 nucleus, and has a warped gas and dust disk which might be caused by an interaction with a close companion galaxy (e.g., Krips et al. 2005). A black hole mass of $\sim 5 \times 10^{7} \mathcal{M}_{\odot}$ is implied by $\sigma_{\mathrm{s}}=157 \mathrm{~km} \mathrm{~s}^{-1}$ (Ho et al. 1997), and a nuclear 

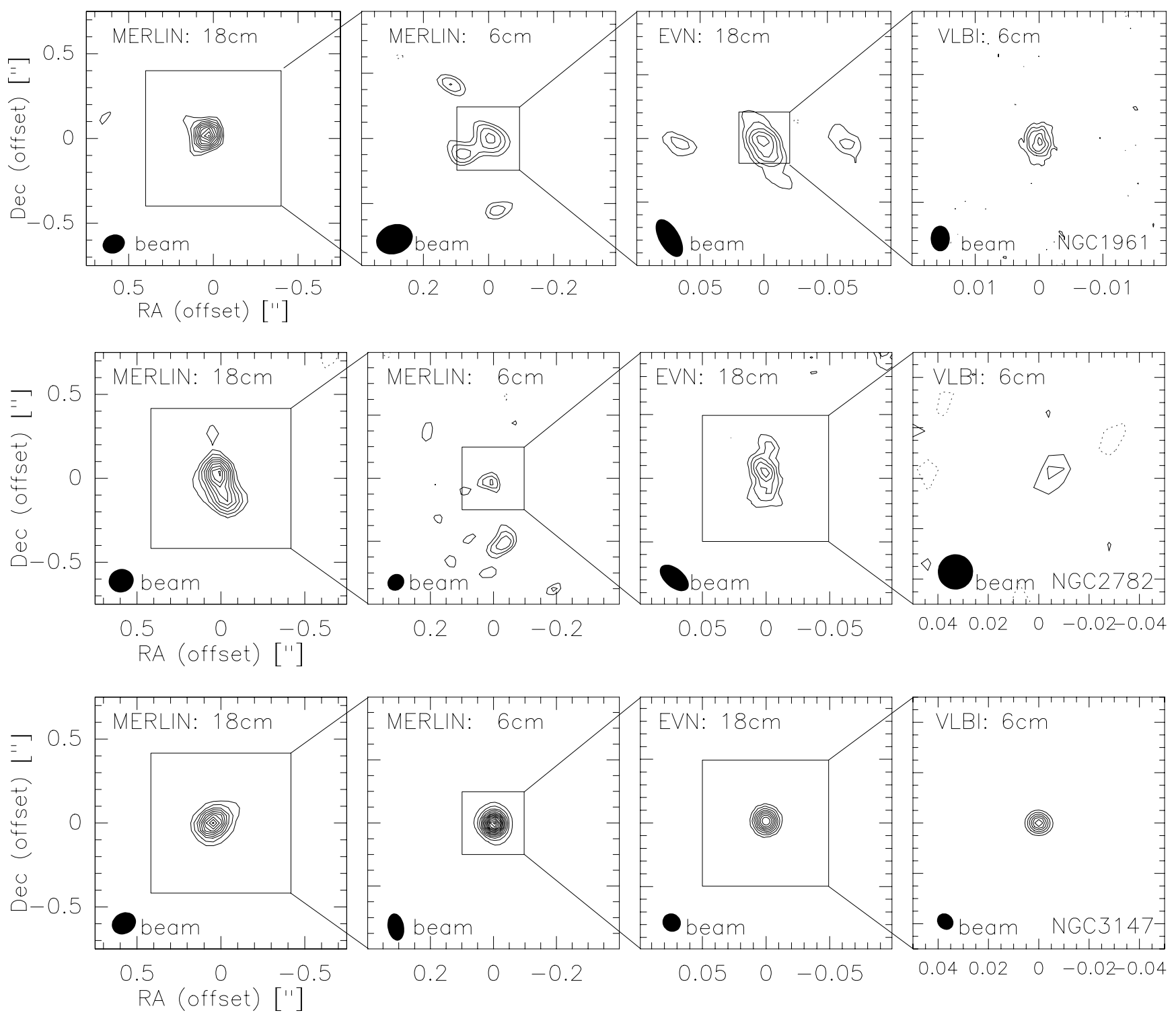

Fig. 1. MERLIN (2 left columns) and EVN/VLBI (two right columns) of the $18 \mathrm{~cm}$ and $6 \mathrm{~cm}$ continuum emission in NGC 1961 (upper panels), NGC 2782 (middle panels) and NGC 3147 (lower panels). The respective synthesized beams are shown in the lower left part of each map. Contour levels for MERLIN-18 cm (NGC 1961; upper left): $(3 \sigma=) 0.57$ to 2.28 mJy/beam in steps of $1 \sigma$; for MERLIN-6 cm (upper left middle): ( $3 \sigma=) 0.39$ to $0.78 \mathrm{mJy} / \mathrm{beam}$ in steps of $1 \sigma$; EVN-18 cm (upper right middle): $(3 \sigma=) 0.21$ to $0.49 \mathrm{mJy} / \mathrm{beam}$ in steps of $1 \sigma$; EVN-6 cm (upper right): $(3 \sigma=) 0.14$ to $0.61 \mathrm{mJy} / \mathrm{beam}$ in steps of $1 \sigma$; MERLIN-18 cm (NGC 2782; middle left): $(3 \sigma=) 0.45$ to $1.7 \mathrm{mJy} / \mathrm{beam}$ in steps of $1 \sigma$, for MERLIN$6 \mathrm{~cm}$ (middle left middle): $(3 \sigma=) 0.39$ to $0.78 \mathrm{mJy} / \mathrm{beam}$ in steps of $1 \sigma$, for EVN-18 cm (middle right middle): $(3 \sigma=) 0.18$ to $0.36 \mathrm{mJy} / \mathrm{beam}$ in steps of $1 \sigma$; for EVN-6 cm (middle left): $(2 \sigma=) 0.2$ to $0.3 \mathrm{mJy} / \mathrm{beam}$ in steps of $1 \sigma$. MERLIN-18 cm (NGC 3147 ; lower left): $(5 \sigma=) 0.75$ to $7.5 \mathrm{mJy} / \mathrm{beam}$ in steps of $5 \sigma$, for MERLIN-6 cm (lower left middle): $(3 \sigma=) 0.72$ to $10.1 \mathrm{mJy} / \mathrm{beam}$ in steps of $5 \sigma$, for EVN-18 cm (lower right middle): $(3 \sigma=) 0.65$ to $5.2 \mathrm{mJy} / \mathrm{beam}$ in steps of $5 \sigma$; for EVN-6 cm (lower left): $(5 \sigma=) 1.4$ to $8.3 \mathrm{mJy} / \mathrm{beam}$ in steps of $5 \sigma$. Negative contours, if visible, correspond to $3 \sigma$.

bolometric luminosity of $\sim 10^{41} \mathrm{erg} \mathrm{s}^{-1}$ (Table 7) implies a subEddington system with $L_{\mathrm{bol}} / L_{\mathrm{ed}} \simeq 10^{-5}-10^{-4}$.

Similar to NGC 3147 , NGC 3718 is among the strongest radio continuum sources in our survey (Table 4). It shows extended emission on MERLIN $18 \mathrm{~cm}$ scales and signs of a quite compact $\left(\sim 0.5^{\prime \prime}=34 \mathrm{pc}\right) 4 \sigma$ jet in a northwest direction (Fig. 2$)$. This is supported by VLA maps that also reveal extended emission on large scales (Condon 1987). There are some weak indications for extended emission from inspection of the $6 \mathrm{~cm}$ maps and comparison of the peak and integrated flux densities. Moreover, the EVN $6 \mathrm{~cm}$ map reveals a slight extension but, in contrast to the MERLIN $18 \mathrm{~cm}$ map, in a westward direction, which we therefore do not identify as the same jet. The flux densities from
MERLIN and EVN are identical, indicating that there is no emission extended on intermediate scales in NGC 3718 other than the small-scale jet. Our fluxes are in good agreement with those obtained in VLBA observations by Nagar et al. (2002). The spectral indices are very consistent for the MERLIN and EVN data and are derived to be 0.4 , describing an inverted spectrum.

\subsubsection{NGC 4579}

NGC 4579 is a SAB(rs)b galaxy at a distance of $20 \mathrm{Mpc}$ with a Seyfert type 1.9 and/or LINER type 1.9 nucleus. The black hole mass is $\sim 10^{8} \mathcal{M}_{\odot}$ assuming $\sigma_{\mathrm{s}}=185 \mathrm{~km} \mathrm{~s}^{-1}$ (McElroy 1995). Ho et al. (1999) determine the nuclear bolometric luminosity to 

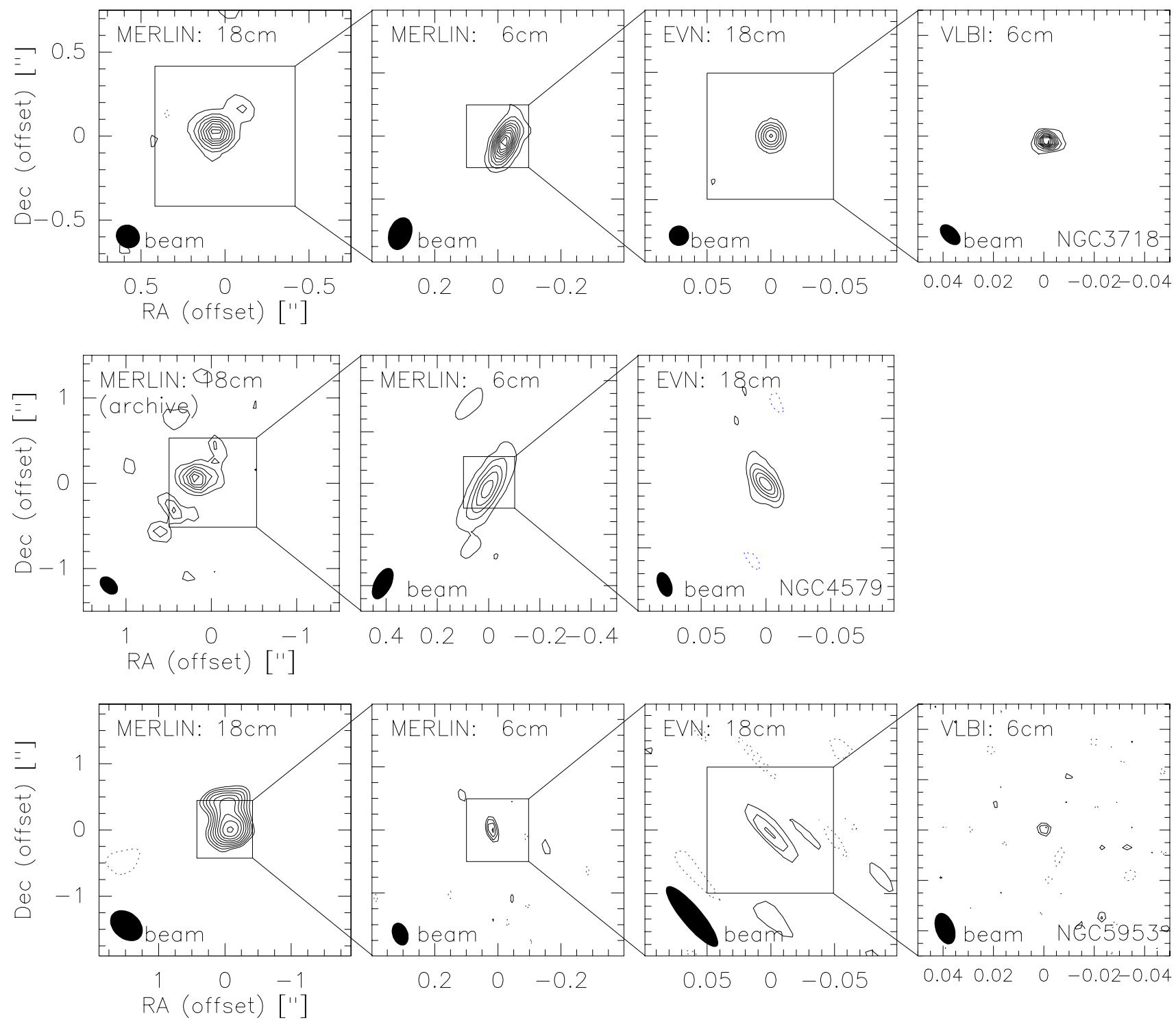

Fig. 2. MERLIN ( 2 images on the left respectively) and EVN ( 2 images on the right respectively) of the $18 \mathrm{~cm}$ and $6 \mathrm{~cm}$ continuum emission in NGC 3718 (upper panel), NGC 4579 (middle panel) and NGC 5953 (lower panel). The respective synthesized beams are shown in the lower left corner of each map. MERLIN-18 cm (NGC 3718; upper left): $(5 \sigma=) 0.64$ to 5.1 mJy/beam in steps of $5 \sigma$, for MERLIN-6 cm (upper left middle): $(5 \sigma=) 0.5$ to $5.0 \mathrm{mJy} / \mathrm{beam}$ in steps of $5 \sigma$, for EVN-18 cm (upper right middle): $(5 \sigma=) 0.75$ to $4.5 \mathrm{mJy} /$ beam in steps of $5 \sigma$; for EVN-6 cm (upper right): $(5 \sigma=) 0.55$ to $5.0 \mathrm{mJy} / \mathrm{beam}$ in steps of $5 \sigma$; Contour levels for MERLIN-6 cm (NGC 4579; middle left middle): $(5 \sigma=) 1.0$ to $15.0 \mathrm{mJy} / \mathrm{beam}$ in steps of $10 \sigma$, for EVN-18 cm (middle right middle): $(3 \sigma=) 3.3$ to $16.5 \mathrm{mJy} / \mathrm{beam}$ in steps of $3 \sigma$; MERLIN-18 cm (NGC 5953 ; lower left): $(3 \sigma=) 0.48$ to $1.5 \mathrm{mJy} /$ beam in steps of $1 \sigma$, for MERLIN-6 cm (lower left middle): $(3 \sigma=) 0.74$ to $1.7 \mathrm{mJy} / \mathrm{beam}$ in steps of $1 \sigma$, for EVN-18 cm (lower right middle): $(2 \sigma=) 0.12$ to $0.24 \mathrm{mJy} / \mathrm{beam}$ in steps of $1 \sigma$; for EVN-6 cm (lower right): $(2 \sigma=) 0.2$ to $0.4 \mathrm{mJy} /$ beam in steps of $1 \sigma$. Negative contours, if visible, correspond to $3 \sigma$.

be $\sim 10^{42} \mathrm{erg} \mathrm{s}^{-1}$. This gives a value of $\sim 10^{-4}$ for $L_{\mathrm{bol}} / L_{\mathrm{ed}}$ indicating a sub-Eddington system.

NGC 4579 was already observed with MERLIN at $18 \mathrm{~cm}$ (PI: N.Nagar, from the MERLIN archive) and with the VLBA at $18 \mathrm{~cm}, 13 \mathrm{~cm}, 6 \mathrm{~cm}$ and $4 \mathrm{~cm}$ (Ulvestad \& Ho 2001). We thus conducted only complementary observations with MERLIN at $6 \mathrm{~cm}$ and EVN at $18 \mathrm{~cm}$ (Fig. 2 and Table 4). In the VLBA maps from Ulvestad \& Ho (2001), no extended emission can be seen, while VLA maps from Ho \& Ulvestad (1999) at $20 \mathrm{~cm}$ and $6 \mathrm{~cm}$ show a jet-like extension of $\sim 3^{\prime \prime}$ to the northwest. Our $18 \mathrm{~cm}$ and $6 \mathrm{~cm}$ maps do not show any jet-like feature on sub-arcsecond scales, but the difference between peak flux and integrated flux still indicates extended emission. Furthermore, the deconvolved size at $18 \mathrm{~cm}$ is slightly larger than the beam, supporting the presence of extended emission. Falcke et al. (2001) report variability of the radio flux at $15 \mathrm{GHz}$ on timescales of $1-3$ years. We too find a difference of $~ 10 \%$ between the EVN and VLBA $18 \mathrm{~cm}$ flux densities. Thus, the determination of the spectral index must be made and interpreted with caution since not all fluxes were measured simultaneously. This might explain the large discrepancy between the MERLIN $18 \mathrm{~cm}$ flux and that from the EVN $18 \mathrm{~cm}$ data, which is higher by a factor of $2-3$.

\subsubsection{NGC 5953}

NGC 5953 is characterised as a SAa pec galaxy at a distance of $26 \mathrm{Mpc}$ hosting a Seyfert type 2 nucleus. It is also known 

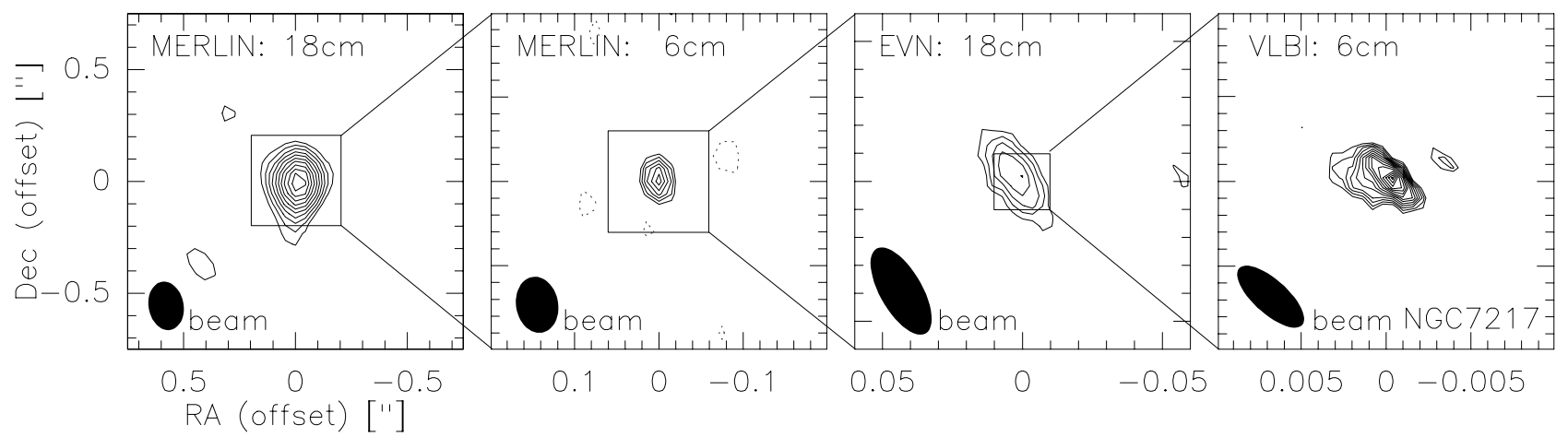

Fig. 3. MERLIN ( 2 images on the left) and EVN ( 2 images on the right) of the $18 \mathrm{~cm}$ and $6 \mathrm{~cm}$ continuum emission in NGC 7217. The respective synthesized beams are shown in the lower middle part of each map. Contour levels for MERLIN- $18 \mathrm{~cm}(l e f t):(3 \sigma=) 0.36$ to $1.4 \mathrm{mJy} / \mathrm{beam}$ in steps of $1 \sigma$, for MERLIN-6 cm (left middle): $(3 \sigma=) 1.5$ to $4.0 \mathrm{mJy} / \mathrm{beam}$ in steps of $1 \sigma$, for EVN-18 cm (right middle): $(3 \sigma=) 0.21$ to $0.5 \mathrm{mJy} / \mathrm{beam}$ in steps of $1 \sigma$; for EVN-6 cm (right): $(3 \sigma=) 0.2$ to $0.6 \mathrm{mJy} / \mathrm{beam}$ in steps of $1 \sigma$. Negative contours, if visible, correspond to $3 \sigma$.

Table 5. Spectral indices of the respective sources. $S_{v} \propto v^{\alpha} .{ }^{a}$ taken from Ulvestad \& Ho (2001).

\begin{tabular}{ccccc}
\hline \hline Name & $\begin{array}{c}\alpha_{113}^{230} \\
(\mathrm{PdBI})\end{array}$ & $\begin{array}{c}\alpha_{1.7}^{5} \\
(\text { MERLIN })\end{array}$ & $\begin{array}{c}\alpha_{1.6}^{5} \\
(\mathrm{EVN})\end{array}$ & $\begin{array}{c}\alpha_{1.7}^{5} \\
(\mathrm{VLBA})^{a}\end{array}$ \\
\hline NGC 1961 & -0.4 & $-0.3 \pm 0.3$ & $-0.3 \pm 0.2$ & - \\
NGC 2782 & - & $-0.6 \pm 0.3$ & $\leq-1.0$ & - \\
jet & - & $-0.2 \pm 0.6$ & - & - \\
NGC 3147 & -0.3 & $0.14 \pm 0.03$ & $0.39 \pm 0.06$ & $0.2 \pm 0.1$ \\
NGC 3718 & $0.0 \pm 0.2$ & $0.4 \pm 0.1$ & $0.37 \pm 0.06$ & - \\
NGC 4579 & 0.1 & $1.12 \pm 0.02$ & $0.09 \pm 0.04$ & $0.2 \pm 0.1$ \\
NGC 5953 & - & $-1.2 \pm 0.5$ & - & - \\
NGC 7217 & - & $0.6 \pm 0.1$ & $0.8 \pm 0.2$ & - \\
\hline
\end{tabular}

to interact with a close companion NGC 5954 (e.g., Iono et al. 2005). The black hole mass of $\sim 7 \times 10^{6} \mathcal{M}_{\odot}\left(\sigma_{\mathrm{s}}=94 \mathrm{~km} \mathrm{~s}^{-1}\right.$; McElroy 1995) is the smallest one in our sample. Woo \& Urry (2002) find a surprisingly high nuclear bolometric luminosity of $\sim 10^{44} \mathrm{erg} \mathrm{s}^{-1}$ but their estimate is based on fluxes taken over very large apertures. No X-ray luminosity has been published yet. Thus, any conclusion about its accretion efficiency remains speculative.

NGC 5953 turns out to be the weakest radio source in our sample (Table 4). It is clearly detected in the MERLIN $18 \mathrm{~cm}$ and $6 \mathrm{~cm}$ maps, while it remains undetected in the EVN maps (Fig. 2). We find $4 \sigma$ peaks in the latter at the position of the nucleus. The MERLIN $18 \mathrm{~cm}$ data show a strong jet to the north, which is slightly resolved with the MERLIN beam $\left(\sim 0.3^{\prime \prime} \equiv\right.$ $41 \mathrm{pc}$ ). A steep spectral index for the core is derived from the MERLIN data, which might be biased by resolution effects in the MERLIN $6 \mathrm{~cm}$ map. VLA maps (e.g., Condon et al. 1990; Iono et al. 2005) also indicate extended emission on large angular scales, but the emission is somewhat smeared between the two interacting systems in the VLA beam.

\subsubsection{NGC 7217}

NGC 7217 contains a LINER type 2 nucleus in an (R)SAB(rs)a host galaxy at a distance of $13 \mathrm{Mpc}$. It has a black hole mass of $\sim 3 \times 10^{7} \mathcal{M}_{\odot}$ assuming $\sigma_{\mathrm{s}}=132 \mathrm{~km} \mathrm{~s}^{-1}$ (McElroy 1995) and an estimated nuclear bolometric luminosity of $\sim 10^{40} \mathrm{erg} \mathrm{s}^{-1}$ (Table 7). Both values result in $L_{\mathrm{bol}} / L_{\mathrm{ed}} \simeq 10^{-6}-10^{-5}$. Thus, NGC 7217 is also a sub-Eddington system.
Table 6. Brightness temperatures estimated with the formula given in Condon et al. (1982). The frequency $(v)$, the flux density $\left(S_{v}\right)$ and the deconvolved size $(\theta)$ as an upper limit are taken from Table 4.

\begin{tabular}{ccccc}
\hline \hline Name & $\begin{array}{c}v \\
{[\mathrm{GHz}]}\end{array}$ & $\begin{array}{c}S_{v} \\
{[\mathrm{mJy}]}\end{array}$ & $\begin{array}{c}\theta \\
{[\mathrm{mas}]}\end{array}$ & $\begin{array}{c}T_{\mathrm{b}} \\
{[\mathrm{K}]}\end{array}$ \\
\hline NGC 1961 & 5.0 & 0.9 & $\leq 1$ & $\geq 4 \times 10^{7}$ \\
NGC 2782 & 1.6 & 1.2 & $\leq 10$ & $\geq 2 \times 10^{6}$ \\
NGC 3147 & 5.0 & 10.1 & $\leq 4$ & $\geq 3 \times 10^{7}$ \\
NGC 3718 & 5.0 & 7.1 & $\leq 1$ & $\geq 3 \times 10^{8}$ \\
NGC 4579 $^{a}$ & 5.0 & 22.8 & $\leq 4$ & $\geq 7 \times 10^{8}$ \\
NGC 5953 & 5.0 & 0.5 & $\leq 50$ & $\geq 1 \times 10^{4}$ \\
NGC 7217 & 5.0 & 1.2 & $\leq 3$ & $\gtrsim 7 \times 10^{6}$ \\
\hline
\end{tabular}

${ }^{a}$ Taken from Ulvestad \& Ho (2001).

The MERLIN $18 \mathrm{~cm}$ map (Fig. 3 and Table 4) clearly shows an extended component with an elongation to the south, although NGC 7217 appears to be unresolved on VLA scales. The MERLIN $6 \mathrm{~cm}$ and EVN $18 \mathrm{~cm}$ component remains pointlike, however, while the EVN $6 \mathrm{~cm}$ map reveals an extended component but in an eastern direction different from that in the MERLIN $18 \mathrm{~cm}$ map. This apparent inconsistency might be a result of the different sensitivity levels and angular resolutions obtained by the respective observations. The MERLIN and EVN spectra are the most strongly inverted in our sample.

\section{Nature of the radio emission: thermal or non-thermal?}

Based on the equation given by Condon et al. (1982) and taking the deconvolved source size as an upper limit for the actual source size and the flux density at $6 \mathrm{~cm}$ from Table 4 (except for NGC 2782), we can estimate lower limits for the brightness temperature in each of the seven galaxies. For most of them, the brightness temperatures are high enough $\left(T_{\mathrm{b}} \gtrsim 10^{8} \mathrm{~K}\right)$ to be consistent with synchrotron emission given that the assumed source sizes are only upper limits. For NGC 3147, for instance, Anderson et al. (2004) derive a source size of $\lesssim 1$ mas, increasing the temperature determined from our data to $\sim 4 \times 10^{8} \mathrm{~K}$. Given the loose upper limit on the source size for NGC 2782, its temperature might also be compatible with synchrotron emission. However, the lower limit on $T_{\mathrm{b}}$ virtually excludes the possibility that the radio emission in the central \pm 10 mas of NGC 2782 is coming from star formation, which typically produces $\leq 10^{5} \mathrm{~K}$, 
Table 7. Black hole masses, bolometric and Eddington luminosities for the seven galaxies.

\begin{tabular}{|c|c|c|c|c|c|c|c|c|c|}
\hline NGC & $\begin{array}{c}\mathrm{D}^{l} \\
{[\mathrm{Mpc}]}\end{array}$ & $\begin{array}{c}M_{\mathrm{bh}} \\
{\left[10^{8} \mathcal{M}_{\odot}\right]}\end{array}$ & $\begin{array}{c}\log \left(L_{5.0}^{\mathrm{VLBI}}\right) \\
{\left[\operatorname{erg~s}^{-1}\right]^{j}}\end{array}$ & $\begin{array}{l}\log \left(L_{5.0}^{\mathrm{VLA}}\right) \\
{\left[\operatorname{erg~s}^{-1}\right]^{m}}\end{array}$ & $\begin{array}{l}\log \left(L_{\mathrm{x}}\right)^{n} \\
{\left[\mathrm{erg} \mathrm{s}^{-1} \text { ] }\right.}\end{array}$ & $\begin{array}{l}\log \left(L_{\mathrm{bol}}\right) \\
{\left[\mathrm{erg} \mathrm{s}^{-1}\right]}\end{array}$ & $\begin{array}{l}\log \left(L_{\text {ed }}\right)^{i} \\
{\left[\mathrm{erg} \mathrm{s}^{-1}\right]} \\
\end{array}$ & $\begin{array}{l}\log \left(L_{\mathrm{bol}} / L_{\mathrm{ed}}\right) \\
\text { range }\end{array}$ & $\log \left(L_{5.0}^{\mathrm{obs}} / L_{\mathrm{x}}\right)$ \\
\hline 1961 & 52 & $3.4^{a}$ & 37.16 & $<38.02$ & $40.56^{c}$ & $\sim 41^{g}$ & 46.6 & {$[-5,-6]$} & -4.23 \\
\hline 2782 & 35 & $0.4^{a}$ & 36.34 & 38.02 & $40.11^{c}$ & $\sim 41^{g}$ & 45.7 & {$[-4,-5]$} & -3.77 \\
\hline 3147 & 38 & $4.2^{a}$ & 37.91 & 38.23 & $41.51^{d}$ & $\sim 42.4^{g}$ & 46.7 & {$[-4,-5]$} & -3.62 \\
\hline 3718 & 13 & $0.5^{a, b}$ & 36.86 & 37.60 & $40.59^{e}$ & $\sim 41^{g}$ & 45.8 & {$[-4,-5]$} & -4.56 \\
\hline 4579 & 20 & $1.0^{a}$ & 37.74 & 38.10 & $41.10^{d}$ & $42.0^{k}$ & 46.1 & -4.1 & -4.04 \\
\hline 5953 & 26 & $0.07^{a}$ & $\leq 36.21$ & 37.25 & - & $44.1^{h}$ & 44.9 & -0.9 & $\leq-7.01$ \\
\hline 7217 & 13 & $0.3^{a}$ & 36.08 & 36.38 & $39.63^{f}$ & $\sim 40^{g}$ & 45.6 & {$[-5,-6]$} & -4.56 \\
\hline
\end{tabular}

${ }^{a}$ Assuming $M_{\mathrm{bh}}=(1.3 \pm 0.1) \times 10^{8} \mathcal{M}_{\odot} \times\left(\sigma_{\mathrm{s}} / 200\left[\mathrm{~km} \mathrm{~s}^{-1}\right]\right)^{4.0 \pm 0.3}$ from Tremaine et al. (2002) with $\sigma_{\mathrm{s}}$ taken from McElroy et al. (1995). ${ }^{b} \sigma_{\mathrm{s}}$ taken from Ho et al. (1997) and assuming $\sigma_{\mathrm{s}}=F W H M([\mathrm{OIII}]) / \sqrt{8 \ln 2}=F W H M([\mathrm{~N} \mathrm{II}]) / \sqrt{8 \ln 2} .{ }^{c}$ Taken from Roberts \& Warwick (2000). ${ }^{d}$ Taken from Ulvestad \& Ho (2001). ${ }^{e}$ Taken from Fabbiano et al. (1992). ${ }^{f}$ Taken from Terashima et al. (2002). ${ }^{g}$ Assuming $L_{\mathrm{bol}}=6.7 \times L_{\mathrm{x}}(2-10 \mathrm{keV})$ from Ulvestad \& Ho (2001); as simplification and since we were interested only in a rough estimate of the bolometric luminosity, we assumed $L_{\mathrm{x}}(2-10 \mathrm{keV}) \approx L_{\mathrm{x}}(0.2-4 \mathrm{keV})$ allowing for half an order of magnitude uncertainty. ${ }^{h}$ Taken from Woo \& Urry (2002). ${ }^{i}$ Taking the standard Eddington equation. ${ }^{j}$ Assuming $L_{5.0}=v_{5.0} \times 4 \pi D^{2} S_{v}$ where $S_{v}$ is the integrated flux density from Table 4. ${ }^{k}$ Ho et al. (1999). ${ }^{l}$ Derived via $D \simeq V_{\text {hel }} / 74 \mathrm{Mpc} .{ }^{m}$ Extrapolated from $15 \mathrm{GHz}$ VLA observations by Nagar et al. (2005) except for NGC 2782, NGC 5953 and NGC 7217 which were taken from 1.4 GHz VLA observations done by Becker et al. (1995), Condon et al. (1996) or Iono et al. (2005). ${ }^{n}$ We corrected the X-ray luminosities to match the distances used in this paper, if significantly different luminosity distances were assumed in the X-ray data papers.

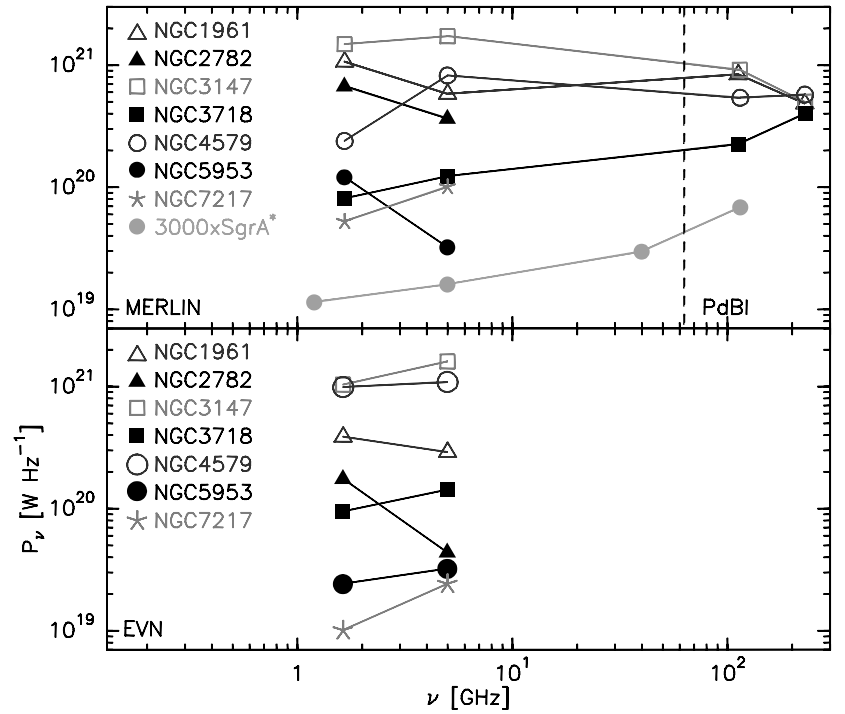

Fig. 4. Spectral energy distribution from the MERLIN/PdBI (upper panel) and EVN data (lower panel). The dashed line in the upper panel separates the MERLIN from the PdBI data. Values for SgrA ${ }^{\star}$ were taken from Falcke et al. (1998) and Zhao et al. (2001).

supporting the presence of synchrotron (self-absorbed) emission from an optically hidden AGN.

The situation for NGC 5953 and NGC 7217 might be different. Although NGC 5953 has a very high upper limit on the source size, its brightness temperature would only go up to $\sim 10^{7} \mathrm{~K}$ when taking a source size of 1 mas, probably still too low to agree with synchrotron self-absorption from a compact source but also too high for star formation. However, optically thin synchrotron emission from an (extended) jet might not disagree with the low brightness temperature; this would also fit to the steep radio spectrum found in NGC 5953. The continuum emission in NGC 7217 appears already slightly resolved in the VLBI $6 \mathrm{~cm}$ map, leaving little leverage to increase the true brightness temperature to $10^{8} \mathrm{~K}$ excluding thus a self-absorbed compact synchrotron source. In contrast to NGC 5953, the inverted radio spectra of NGC 7217 are not in agreement with optically thin synchrotron emission. Alternatively, thermal free-free emission or electron scattered synchrotron emission may be the mechanism responsible for the continuum similar to NGC 1068 (e.g., Roy et al. 1998; Gallimore et al. 1997, 2004; Krips et al. 2006). Although we see no evidence that starbursts dominate the centers of our LLAGN, the larger VLA fluxes imply the presence of larger scale diffuse emission that might be produced by starbursts.

\section{The fundamental plane}

A highly significant relationship linking X-ray luminosity, black hole mass, and radio luminosity has been found to hold for many black hole systems, including both X-ray binaries and active galaxies (e.g., Merloni et al. 2003; Falcke et al. 2004). In Figs. 5 and 6, the VLBI luminosities at $5 \mathrm{GHz}\left(L_{5.0}\right)$ are compared with published X-ray luminosities (Fabbiano et al. 1992; Roberts \& Warwick 2000; Terashima et al. 2002) and black hole masses (McElroy et al. 1995) for six of our objects, along with literature data for $\sim 30$ LINER and Seyfert galaxies taken from Terashima et al. (2002), Merloni et al. (2003) and Nagar et al. (2005). In contrast to Merloni et al. (2003), and Falcke et al. 2004, 50\% of the plotted radio fluxes were obtained with the VLBA or VLBI, i.e., at higher angular resolution, reducing the contamination of nuclear emission by extended components such as (large-scale) jets or star formation.

We find that our new data are overall in good agreement with the existing data and lie within the same empirical "fundamental plane" derived by Merloni et al. (2003) and Falcke et al. (2004), even in those cases for which we used a crude estimate for the hard X-ray luminosity. This relation has been interpreted theoretically in terms of a disk-jet coupling, as suggested by Heinz \& Sunyaev (2003), radiatively inefficient accretion flows, or a combination of both. Radiatively efficient accretion flows can be almost completely ruled out for these sub-Eddington systems (Merloni et al. 2003). The larger scatter in the literature data compared to Heinz \& Di Matteo (2003) and Falcke et al. (2004) might be caused by the uncertainty of the assumed spectral indices that were used to extrapolate the $5 \mathrm{GHz}$ fluxes from the published $15 \mathrm{GHz}$ data.

Within our sample, NGC 7217 seems to have the largest offset from the fundamental plane. As mentioned in Sect. 4, 

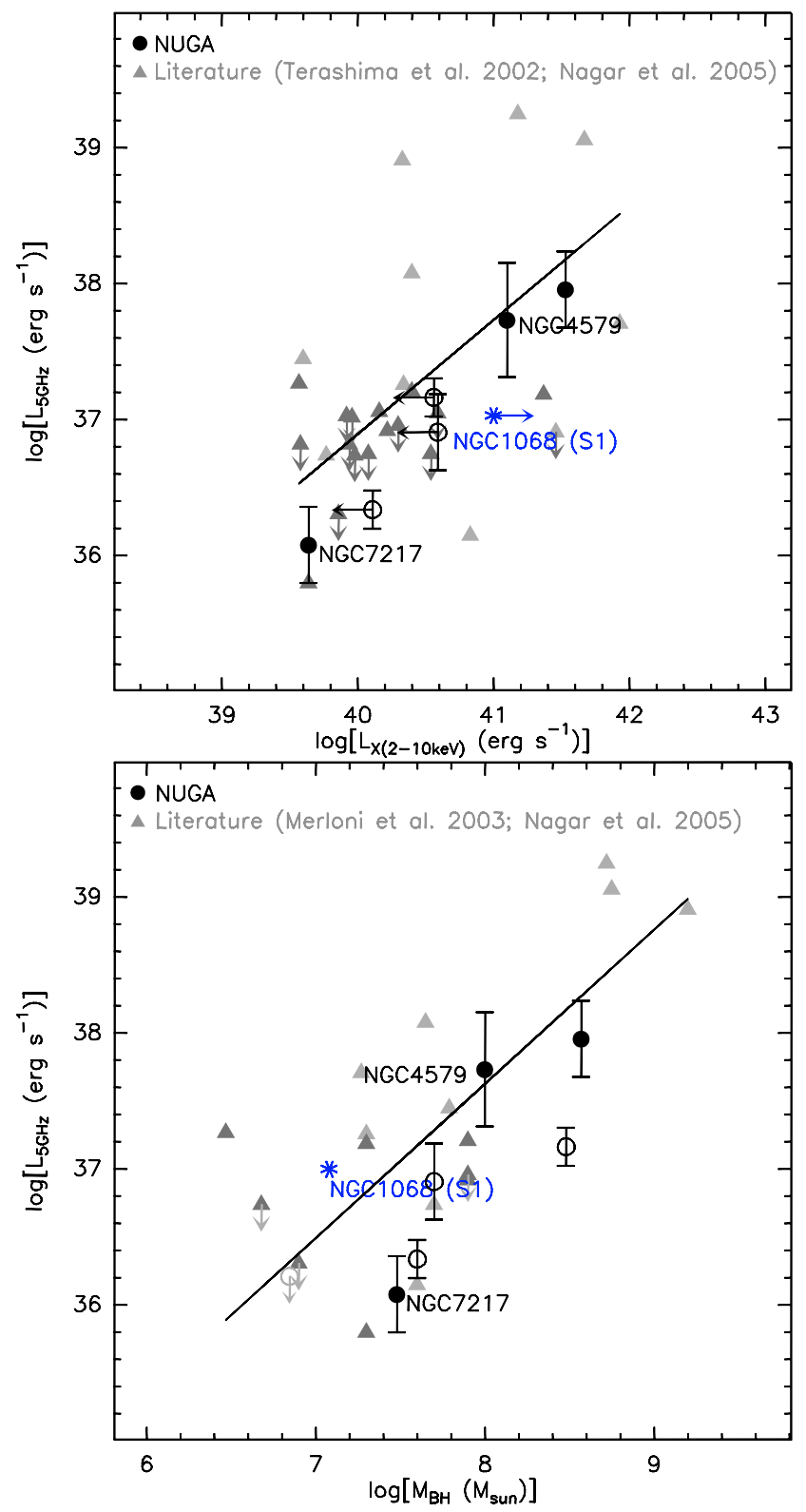

Fig. 5. Comparison of radio $(5 \mathrm{GHz})$ with $\mathrm{X}$-ray luminosities (upper panel) and with black hole mass (lower panel:). We extrapolated the $15 \mathrm{GHz}$ literature data (triangles) to $5 \mathrm{GHz}$ where necessary using the spectral indices indicated in Merloni et al. (2003); this might cause a larger scatter of the data because of their larger uncertainty. The filled light grey triangles represent VLA data while the filled dark grey triangles are taken with the VLBA. For those galaxies from our sample, which lack published hard (2-10 keV) X-ray luminosities (open black circles $)$, we assumed $L_{\mathrm{X}}(0.2-4 \mathrm{keV}) \gtrsim L_{\mathrm{X}}(2-10 \mathrm{keV})$ as simplification (see text for discussion). The open grey circle represents NGC 5953. The literature values are taken from Terashima et al. (2002; X-ray), Nagar et al. (2005; VLBA or VLA data at $15 \mathrm{GHz}$ ) and Merloni et al. $\left(2003 ; M_{\mathrm{BH}}\right)$. Errors of the radio luminosities are from the EVN $5.0 \mathrm{GHz}$ data. The radio data (blue star) from NGC 1068 refer to component $\mathrm{S} 1$ which is supposed to be the AGN and are taken from Gallimore et al. (2004), while the black hole mass is from Huré (2002) and Lodato $\&$ Bertin (2003). The X-ray luminosity is adopted from Merloni et al. (2003) but has to be regarded as a lower limit due to a possible heavy obscuration of the AGN in NGC 1068.

NGC 7217 does not appear to be consistent with (direct) synchrotron emission. It is unclear at this point whether its emission is produced by electron-scattered synchrotron emission, as might

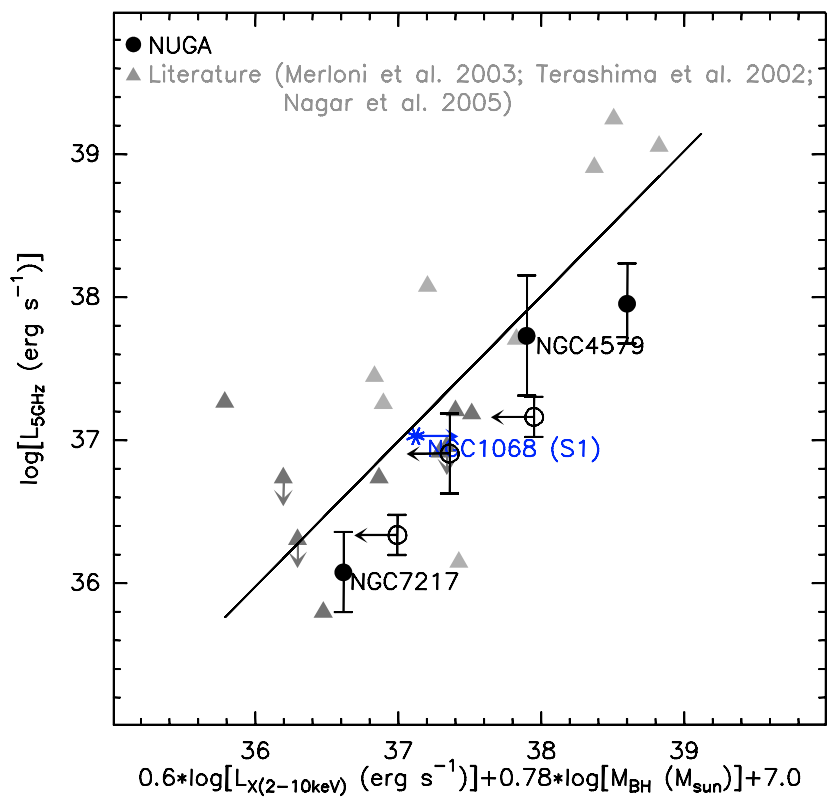

Fig. 6. Comparison of radio fluxes with a projection of the "fundamental plane" derived by Merloni et al. (2003). See caption of Fig. 5 for more details on data.

be the case in NGC 1068 (e.g., Roy et al. 1998; Gallimore et al. 1997, 2004; Krips et al. 2006), or by thermal free-free radiation. The large uncertainty of the X-ray luminosity in NGC 1068 due to the heavy obscuration of its AGN and its large variablity (see discussion in Gallimore et al. 2004) also questions the compatibility of NGC 1068 with the fundamental plane (Figs. 5 and 6), when the VLBA flux of the AGN (component S1) is plotted instead of the lower angular resolution VLA flux adopted by Merloni et al. (2003). The latter is certainly contaminated by emission from the extended parts of the jet.

\section{Summary and discussion}

This paper presents the first results of snapshot radio observations with MERLIN and EVN of a sample of seven LLAGN taken from the NUGA survey. Besides compact emission, smallscale extended emission in the form of jets is also found in these weak radio sources. The radio fluxes decrease significantly with increasing angular resolution at the respective wavelength, indicating the presence of extended and diffuse emission. With one exception (NGC 5953), the radio core components have flat to inverted spectra within the errors. A comparison of the derived radio luminosities to the black hole mass, estimated via the respective stellar velocity dispersions, and the X-ray luminosities suggests correlations among these quantities even at high angular resolution, thus supporting the existence of a fundamental plane. However, as NGC 1068 and NGC 7217 demonstrate, this fundamental plane could be violated if the radio emission is produced not by direct synchrotron processes, but rather by electron scattered synchrotron or free-free emission, even though the latter are also expected to be closely connected to X-ray activity. Whether or not such discrepancies reflect substantially different activity mechanisms in NGC 1068, NGC 7217, and the remaining LLAGN in our sample has to be further investigated. This result emphasizes the need for high angular resolution observations of the radio emission in these sources to verify the validity of the fundamental plane and test its limits. 
Acknowledgements. Part of this work was supported by the German Sonderforschungsbereich, SFB, project number 494. SL acknowledges support by DGI Grant AYA 2002-03338 and Junta de Andalucía'. We thank the referee, N. Nagar, for very useful and careful comments that helped to improve the quality of the paper. We also want to thank Dr. Anita Richards, Dr. Tom Muxlow, Dr. Enno Middelberg and Dr. Walter Alef for their support during the observations and data reduction. AJB acknowledges support from the National Radio Astronomy Observatory, which is operated by Associated Universities, Inc., under cooperative agreement with the National Science Foundation.

\section{References}

Anderson, J. M., \& Ulvestad, J. S. 2005, ApJ, 627, 674

Anderson, J. M., Ulvestad, J. S., \& Ho, L. C. 2004, ApJ, 603, 42

Ball, G. H., Narayan, R., \& Quataert, E. 2001, ApJ, 552, 221

Beasley, A. J., \& Conway, J. E. 1995, in Very Long Baseline Interferometry and the VLBA, ed. J. A. Zensus, P. J. Diamond, \& Napier (San Francisco: ASP), ASP Conf. Ser., 82, 327

Becker, T., \& Duschl, W. J. 1997, A\&A, 328, 95

Becker, R. H., White, R. L., \& Helfand, D. J. 1995, ApJ, 450, 559

Blandford, R. D. 1993, in Astrophysical Jets, ed. D. Burgerella, M. Livio, \& C. P. O'Dea (Cambridge Univ. Press), 15

Blandford, R. D., \& Begelman, M. C. 1999, MNRAS, 303, 1

Braatz, J. A., Henkel, C., Greenhill, L. J., Moran, J. M., \& Wilson, A. S. 2004, ApJ, 617, 29

Combes, F., García-Burillo, S., Boone, F., et al. 2004, A\&A, 414, 857

Condon, J. J. 1987, ApJS, 65, 485

Condon, J. J., Condon, M. A., Gisler, G., \& Puschell, J. J. 1982, ApJ, 252, 102

Condon, J. J., Helou, G., Sanders, D. B., \& Soifer, B. T. 1990, ApJS, 73, 359

Condon, J. J., Anderson, E., \& Broderick, J. J. 1995, AJ, 109, 2318

Condon, J. J., Helou, G., Sanders, D. B., \& Soifer, B. T. 1996, ApJS, 103, 81

Duric, N., \& Seaquest, E. R. 1986, CaJPH, 64, 531

Eckart, A., Schödel, R., \& Straubmeier, Ch. 2004, The Exploration of the Supermassive Black Hole at the center of the Milky Way (manuscript) (Imperial College Press, London), to appear

Fabbiano, G., Kim, D.-W., \& Trinchieri, G. 1992, ApJ, 80, 531

Falcke, H., \& Markoff, S. 2000, A\&A, 362, 113

Falcke, H., Nagar, N. M., Wilson, A. S., \& Ulvestad, J. S. 2000, ApJ, 542, 197

Falcke, H., Lehár, J., Barvainis, R., Nagar, N. M., \& Wilson, A. S. 2001, in ASP Conf. Ser., 224, Probing the Physics of Active Galactic Nuclei, ed. B. M. Peterson, R. W. Pogge, \& R. S. Polidan (San Francisco: ASP), 265

Falcke, H., Körding, E., \& Markoff, S. 2004, A\&A, 414, 895

Ferrarese, L., \& Merritt, D. 2000, ApJ, 539, 9

García-Burillo, S., Combes, F., Hunt, L. K., et al. 2003, A\&A, 407, 485

García-Burillo, S., Combes, F., Schinnerer, E., Boone, F., \& Hunt, L. K. 2005, A\&A, 441, 1011
Gallimore, J. F., Baum, S. A., \& O'Dea, C. P. 1997, Nature, 388, 852 Gallimore, J. F., Baum, S. A., \& O'Dea, Ch. P. 2004, ApJ, 613, 794 Gebhardt, K., Bender, R., Bower, G., et al. 2000, ApJ, 539, 13

Häring, N., \& Rix, H.-W. 2004, ApJ, 604, L89

Ho, L. C. 1999, ApJ, 516, 672

Ho, L. C., \& Ulvestad, J. S. 2001, ApJS, 133, 77

Ho, L. C., Filippenko, A. V., \& Sargent, W. L. W. 1997, ApJS, 112, 315

Hummel, E., van Gorkom, J. H., \& Kontanyi, C. G. 1983, ApJ 267, L5

Huré, J.-M. 2002, A\&A, 395, L21

Iono, D., Yun, M. S., \& Ho, P. T. P. 2005, ApJS, 158, 1

Irwin, J. A., \& Seaquest, E. R. 1988, ApJ, 335, 658

Kaspi, S., Smith, P. S., Netzer, H., et al. 2000, ApJ, 533, 631

Kormendy, J., \& Richstone, D. 1995, ARA\&A, 33, 581

Krips, M., Eckart, A., Neri, R., et al. 2005, A\&A 442, 479

Krips, M., Eckart, A., Neri, R., et al. 2006, A\&A, 446, 113

Lodato, G., \& Bertin, G. 2003, A\&A, 398, 517

Magorrian, J., Tremaine, S., Richstone, D., et al. 1998, AJ, 115, 2285

Marconi, A., \& Hunt, L. K. 2003, ApJ, 589, 21

Merloni, A., Heinz, S., \& di Matteo, T. 2003, MNRAS, 345, 1057

McElroy, D. B. 1995, ApJS, 100, 105

Melia, F., \& Falcke, H. 2001, ARA\&A, 39, 309

van Moorsel, G., Kemball, A., \& Greisen, E. 1996, ASPC, 101, 37

Nagar, N. M., Falcke, H., Wilson, A. S., \& Ulvestad, J. S. 2002, A\&A, 392, 53

Nagar, N. M., Falcke, H., \& Wilson, A. S. 2005, A\&A, 435, 521

Narayan, R., Yi, I., \& Mahadevan, R. 1995, Nature, 374, 623

Nelson, C. H., Green, R. F., Bower, G., Gebhardt, K., \& Weistrop, D. 2004, ApJ, 615,652

Onken, C. A., Ferrarese, L., Merritt, D., et al. 2004, ApJ, 615, 645

Pedlar, A., Kukula, M. J., Longley, D. P. T., et al. 1993, MNRAS 263, 471

Peterson, B. M., Bentz, M. C., Desroches, L.-B., et al. 2005, ApJ, 632, 799

Pringle, J. E. 1993, in Astrophysical Jets, ed. D. Burgerella, M. Livio, \& C. P. O’Dea (Cambridge Univ. Press), 1

Quataert, E. 2003, Astron. Nachr. Suppl., 324, 435

Richstone, D., Ajhar, E. A., Bender, R., et al. 1998, Nature, 395, 14

Roberts, T. P., \& Warwick, R. S. 2000, MNRAS, 315, 98

Roy, A., Colbert, E. J. M., Wilson, A. S., \& Ulvestad, J. S. 1998, ApJ, 504, 147

Saikia, D. J., Pedlar, A., Unger, S. W., \& Axon, D. J. 1994, MNRAS, 270, 46

Shields, G. A., Gebhardt, K., Salviander, S., et al. 2003, ApJ, 583, 124

Shepherd, M. C. 1997, ASPC, 125, 77

Terashima, Y., Iyomoto, N., Ho, L. C., \& Ptak, A. F. 2002, ApJS, 139, 1

Tremaine, S., Gebhardt, K., Bender, R., et al. 2002, ApJ, 574, 740

Ulvestad, J. S., \& Ho, L. C. 2001, ApJ, 562, L133

Ulvestad, J. S., \& Wilson, A. S. 1984, ApJ, 278, 544

Wandel, A. 1999, ApJ, 519, L39

Woo, J.-H., \& Urry, C. M. 2002, ApJ, 579, 530

Yuan, F., Markoff, S., \& Falcke, H. 2002, A\&A, 383, 854

Zhao, J.-H., Bower, G. C., \& Goss, W. M. 2001, ApJ, 547, 29 